\title{
Coffee consumption and the risk of prostate cancer: the Ohsaki Cohort Study
}

\author{
Q Li ${ }^{*}{ }^{1,2}$, M Kakizaki ${ }^{1}$, Y Sugawara ${ }^{1}$, Y Tomata ${ }^{1}$, T Watanabe ${ }^{1}$, Y Nishino ${ }^{3}$ and I Tsuji ${ }^{1}$ \\ ${ }^{1}$ Division of Epidemiology, Department of Public Health and Forensic Medicine, Tohoku University Graduate School of Medicine, \\ Sendai, Japan; ${ }^{2}$ Department of Epidemiology and Health Statistics, School of Public Health, Xi'an Jiaotong University College of \\ Medicine, Xi'an, China and ${ }^{3}$ Division of Cancer Epidemiology and Prevention, Miyagi Cancer Center Research Institute, \\ Natori, Japan
}

Background: Epidemiological evidence regarding the effect of coffee on the incidence of prostate cancer is inconsistent. We aimed to investigate coffee consumption and the risk of prostate cancer risk in a general Japanese population.

Methods: We conducted a prospective cohort study in Ohsaki city, Japan, where 18853 men aged 40-79 years participated in a baseline survey. Coffee consumption was assessed via a validated self-administered questionnaire. During 11 years of follow-up (from January 11995 to December 31, 2005), 318 incident cases of prostate cancer were detected. The Cox proportional hazards regression model was used to calculate the hazard ratios (HRs) and $95 \%$ confidence interval (Cls).

Results: There was a significant inverse association between coffee consumption and the incidence risk of prostate cancer. Compared with those who did not drink coffee, the multivariate adjusted HRs were 0.81 (95\% Cl: 0.61-1.07), 0.73 (95\% Cl: $0.53-$ 1.00), and 0.63 (095\% Cl: 0.39-1.00) for those who drank coffee occasionally, 1-2 cups per day, and $\geqslant 3$ cups per day, respectively, with a $P$ for trend of 0.02 .

Conclusion: This prospective finding from a Japanese population adds evidence that coffee intake is inversely associated with the incidence of prostate cancer.

Prostate cancer is the second most frequently diagnosed cancer and the sixth leading cause of cancer death in men globally (World Health Organization, 2008; Jemal et al, 2011). Recently, the incidence of prostate cancer in Japan has increased rapidly (Matsuda et al, 2012), and it is estimated that by 2020 it will become the most commonly diagnosed cancer in Japanese men (Tabata et al, 2008). Primary prevention of prostate cancer is therefore a critical public health challenge.

Coffee is one of the most frequently consumed beverages in Japan and its popularity continues to increase as the national lifestyle becomes more westernised. Recent national data from Japan have revealed that the average per capita coffee consumption is about $127.1 \mathrm{~g}$ per day (Japan Ministry of Health Labour and Welfare, 2010). Therefore, even small effects of coffee on individuals can have a large effect on public health.

The antioxidant, anti-inflammatory, and anti-carcinogenic activities of coffee constituents suggest that coffee would likely prevent cancer (Cavin et al, 2002; Svilaas et al, 2004; Gomez-Ruiz et al, 2007; Cardenas et al, 2011). In fact, we have observed protective effects of coffee consumption on the risk of liver, oral, pharyngeal, and oesophageal cancers (Shimazu et al, 2005; Naganuma et al, 2008). Coffee drinking is also positively related to the serum levels of total testosterone and sex hormones (Hsieh et al, 1998; Svartberg et al, 2003), which further influence the risk of prostate cancer. However, previous studies of the association between coffee consumption and prostate cancer risk produced inconsistent results. Although earlier prospective studies did not find any such association (Phillips and Snowdon, 1983; Jacobsen et al, 1986; Nomura et al, 1986; Severson et al, 1989; Hsing et al, 1990; Le Marchand et al, 1994; Ellison, 2000; Nilsson et al, 2010), more recent studies including a large cohort in the Health Professionals Follow-up study conducted in USA and a small cohort in UK have reported that consumption of coffee was associated with a lower risk of aggressive (or high Gleason grade)

*Correspondence: Dr Q Li, E-mail: liqiangda@yahoo.com.cn

Received 22 January 2013; revised 17 April 2013; accepted 22 April 2013; published online 14 May 2013

(C) 2013 Cancer Research UK. All rights reserved 0007-0920/13 
prostate cancer (Wilson et al, 2011; Shafique et al, 2012). As few studies have involved Asian populations, we conducted the present prospective study in Japan, where the national lifestyle (including coffee consumption) and the incidence of prostate cancer are different from those in western countries, to further test the association between coffee consumption and total prostate cancer incidence.

\section{MATERIALS AND METHODS}

Study population. We used a prospective study cohort, the Ohsaki cohort, to evaluate the association between coffee consumption and the risk of prostate cancer. The details design and implementation of this population-based prospective cohort study conducted in Ohsaki city, Miyagi Prefecture, northeastern Japan, have been reported previously (Tsuji et al, 1998; Kuriyama et al, 2006; Li et al, 2008; Watanabe et al, 2009). Briefly, between October and December 1994, all National Health Insurance (NHI) beneficiaries aged 40-79 years living in the catchment area of the Ohsaki Public Health Center were delivered a self-administered questionnaire to collect baseline characteristics (including demographic data, medical history, health-related lifestyle factors, and dietary habits) and then followed up. The Ohsaki Public Health Center, a local government agency, provides preventive health care services to the residents of 14 municipalities in Miyagi Prefecture. Among a total of 26481 eligible male participants, 24895 (94\%) responded. We considered the return of self-administered questionnaires signed by the participants to imply their consent to participate in the study. The study protocol was reviewed and approved by the ethics committee of Tohoku University School of Medicine.

We excluded 322 subjects from our cohort study because they had withdrawn before the start of follow-up from 1 January 1995. We also excluded 1558 subjects with a history of cancer, 3957 subjects who failed to supply data on coffee consumption, and 205 subjects who reported extreme daily energy intakes (upper $0.5 \%$ cutoff $3592.18 \mathrm{kcal}$ per day; lower $0.5 \%$ cutoff $383.13 \mathrm{kcal}$ per day) or for whom data on diet were missing. Finally, 18853 subjects remained for analysis. During 11 years of follow-up (to 31 December 2005), 318 incident cases of prostate cancer were identified.

Measurement. We collected baseline data (age, height, weight, smoking and drinking status, physical activity and other lifestyle characteristics) through a structured questionnaire. Dietary habits including coffee drinking were assessed using a valid food frequency questionnaire (FFQ) in which five food consumption frequency categories were used for major foods or food groups. The five categories for drinks such as coffee and tea were: almost never, occasionally, $1-2$ cups per day, $3-4$ cups per day, and $\geqslant 5$ cups per day. The volume of a typical cup of coffee was $150 \mathrm{ml}$ in the study region. In addition, we collected information on whether sugar and/or milk were regularly added to the coffee. We combined the last two categories of coffee consumption into a single category of $\geqslant 3$ cups per day because of the low numbers of individuals in the highest consumption categories. We then classified the subject into four groups (never, occasionally, $1-2$ cups per day, and $\geqslant 3$ cups per day). The validity of this FFQ was subsequently evaluated by calculating Spearman's correlation coefficients for the four 3-day diet records collected during four seasons within the year, and the reproducibility of this FFQ was evaluated from the coefficients of correlation between the two FFQs 1 year apart. The results for coffee showed a reasonably high level of reproducibility $\left(r_{\mathrm{s}}=0.70\right)$ and validity $\left(r_{\mathrm{s}}=0.72\right)$ among our study population (Shimazu et al, 2005). No questions were asked about the type of coffee drunk, the method of brewing or temperature. The volume of a typical cup of coffee in the study area was $150 \mathrm{ml}$.
Follow-up and case ascertainment. We followed the incidence of cancer through a computerised record link to the Miyagi Prefecture Cancer Registry, one of the oldest and most accurate populationbased cancer registries in Japan (Takano and Okuno, 1997; Curado et al, 2007). The relevant cases were abstracted from the medical records of the hospitals by a medical doctor or trained medical records reviewer, except for cases reported to the registry from an institution. The percentage registered by death certificates only (DCO) for prostate cancer was 8\% for men during 1998-2002. We ascertained the death, emigration or loss of NHI qualification of each study subject, and the corresponding date, from the NHI claim history files kept by Miyagi NHI Association. The end point of interest was prostate cancer incidence. According to the International Classification of Diseases for Oncology, 2nd edition (ICD-O-2), prostate cancer incidence was defined as code C61.9. Clinical stage was classified as localised (cancer confined to within the prostate), advanced (extraprostatic or metastatic cancer involving lymph nodes or other organs) or unknown. If clinical stage information was not available, a Gleason score of 7 or less was considered to indicate localised cases, and a score of $\geqslant 8$ was considered to indicate advanced cases. Follow-up of the subjects who had withdrawn from the NHI was discontinued because of logistic limitations. We obtained the baseline information, the NHI claim history, and the cancer registry data in individual files and connected them with the unique ID number of each beneficiary.

Statistical analysis. We counted the person-years of follow-up for each subject from 1 January 1995, until the date of diagnosis of prostate cancer, date of withdrawal from the NHI, date of death, or the end of the study period (31 December 2005), whichever occurred first. A total of 168181 person-years were accumulated. We used the Cox proportional hazards regression model to estimate the hazard ratios (HRs) and 95\% confidence interval (CIs) of prostate cancer incidence according to the consumption of coffee, using the SAS version 9.3 statistical software package (SAS Institute Inc., Cary, NC, USA). Tests of HR linear trend for the different frequencies of coffee consumption were performed by fitting models with the orders of each consumption category as a continuous variable. We conducted further analysis excluding prostate cancer cases diagnosed in the first 2 years to minimise the possibility that diet or lifestyle factors had changed in response to subclinical disease. We also conducted stratified analysis according to the main confounders (such as age, body mass index, smoking status, alcohol drinking status, and stage of prostate cancer) to lessen the possible influence of convincing covariates. Additionally, separate analyses of prostate cancer incidence according to differences in coffee type and tea type were conducted. All reported $P$-values are two-sided, and the cutoff point for statistical significance was $<0.05$.

We considered the following variables to be potential confounders, as they are major risk factors for prostate cancer or correlated with both coffee consumption and prostate cancer incidence (Nilsson et al, 2010; Wilson et al, 2011): age at the baseline (continuous variable), education level (junior high school or lower, high school, college/university or higher), body mass index (BMI, calculated as weight in $\mathrm{kg}$ divided by height in $\mathrm{m}^{2}$ $\left(<18.5,18.5-24.9\right.$ or $\left.\geqslant 25.0 \mathrm{~kg} \mathrm{~m}^{-2}\right)$, time engaged in sports or exercise $(<1,1-2$ or $\geqslant 3 \mathrm{~h}$ per week), marital status (married, or widowed/divorced/single), time spent walking $(<1$ or $\geqslant 1 \mathrm{~h}$ per day), smoking status (never, former, current smoking $<20$ cigarettes per day, or current smoking $\geqslant 20$ cigarettes per day), family history of cancer (yes or no), consumption of tea (never or occasionally, $1-2,3-4$ or $\geqslant 5$ cups per day), job status (employed or unemployed), daily total energy intake (continuous variable), passive smoking (yes or no), alcohol drinking (never, former, current with $<45.6 \mathrm{~g}$ per day ethanol, or current 


\section{Table 1. Baseline characteristics of participants according to the frequency of coffee consumption}

Frequency of coffee consumption

\begin{tabular}{|c|c|c|c|c|c|}
\hline Characteristics & Never $(n=3,637)$ & $\begin{array}{l}\text { Occasionally } \\
(n=6,680)\end{array}$ & $\begin{array}{l}\text { 1-2 Cups per day } \\
(\mathrm{n}=5,959)\end{array}$ & $\begin{array}{c}\geqslant 3 \text { Cups per day } \\
(n=2,577)\end{array}$ & $\boldsymbol{P}$-value \\
\hline Age in years, mean (s.d.), year & $61.5(10.0)$ & $60.1(10.0)$ & $56.8(10.5)$ & $53.4(10.4)$ & $<0.01$ \\
\hline Job status, $n(\%)$ & & & & & $<0.01$ \\
\hline Employed & $1874(69.1 \%)$ & $3972(79.7 \%)$ & $3746(84.0 \%)$ & $1769(89.9 \%)$ & - \\
\hline Unemployed & 839 (30.9\%) & $1,013(20.3 \%)$ & $711(16.0 \%)$ & $198(10.1 \%)$ & - \\
\hline Education level, n (\%) & & & & & $<0.01$ \\
\hline Junior high school or lower & $2344(67.0 \%)$ & $4011(62.2 \%)$ & $3200(55.5 \%)$ & 1207 (48.1\%) & - \\
\hline High school & $942(26.9 \%)$ & $1965(30.5 \%)$ & $2048(35.5 \%)$ & $1023(40.7 \%)$ & - \\
\hline College/university or higher & $213(6.1 \%)$ & 469 (7.3\%) & $522(9.0 \%)$ & $281(11.2 \%)$ & - \\
\hline Marital status, n (\%) & & & & & 0.27 \\
\hline Married & $3025(88.6 \%)$ & $5528(89.2 \%)$ & $4998(89.2 \%)$ & $2121(87.9 \%)$ & - \\
\hline Widowed/divorced/single & $389(11.4 \%)$ & $667(10.8 \%)$ & $606(10.8 \%)$ & $292(12.1 \%)$ & - \\
\hline BMI, n (\%) & & & & & 0.68 \\
\hline$<25.0 \mathrm{~kg} \mathrm{~m}^{-2}$ & $2553(73.8 \%)$ & $4705(73.6 \%)$ & $4264(74.4 \%)$ & $1857(74.5 \%)$ & - \\
\hline$\geqslant 25.0 \mathrm{~kg} \mathrm{~m}^{-2}$ & $908(26.2 \%)$ & $1692(26.4 \%)$ & $1468(25.6 \%)$ & $636(25.5 \%)$ & - \\
\hline Weekly sport duration, $n$ (\%) & & & & & $<0.01$ \\
\hline$\geqslant 3 \mathrm{~h}$ & $538(15.6 \%)$ & $991(16.0 \%)$ & 789 (14.0\%) & $293(12.0 \%)$ & - \\
\hline $1-2 \mathrm{~h}$ & $510(14.8 \%)$ & 987 (15.9\%) & $843(15.0 \%)$ & $346(14.2 \%)$ & - \\
\hline$<1 \mathrm{~h}$ & $2394(69.6 \%)$ & $4233(68.2 \%)$ & $3990(71.0 \%)$ & $1798(73.8 \%)$ & - \\
\hline Walking duration, $n(\%)$ & & & & & $<0.01$ \\
\hline$<1 \mathrm{~h}$ per day & $1448(43.0 \%)$ & $3004(48.2 \%)$ & $2794(49.9 \%)$ & $1282(52.3 \%)$ & \\
\hline$\geqslant 1 \mathrm{~h}$ per day & $1918(57.0 \%)$ & $3224(51.8 \%)$ & $2803(50.1 \%)$ & $1169(47.7 \%)$ & \\
\hline Smoking status, $n(\%)$ & & & & & $<0.01$ \\
\hline Never & $770(22.9 \%)$ & $1406(22.9 \%)$ & 966 (17.5\%) & 290 (12.1\%) & - \\
\hline Former & $1094(32.5 \%)$ & $1761(28.7 \%)$ & $1271(23.0 \%)$ & $371(15.5 \%)$ & - \\
\hline Current, $<20$ cigarettes per day & $631(18.7 \%)$ & $1131(18.4 \%)$ & $1038(18.8 \%)$ & 335 (14.0\%) & - \\
\hline Current, $\geqslant 20$ cigarettes per day & 874 (25.9\%) & $1843(30.0 \%)$ & $2250(40.7 \%)$ & $1405(58.5 \%)$ & - \\
\hline Passive smoking, $n$ (\%) & & & & & $<0.01$ \\
\hline Yes & $1701(46.8 \%)$ & $2919(43.7 \%)$ & $2083(35.0 \%)$ & $619(24.0 \%)$ & - \\
\hline No & $1936(53.2 \%)$ & $3761(56.3 \%)$ & $3876(65.0 \%)$ & $1958(76.0 \%)$ & - \\
\hline Alcohol drinking, $n$ (\%) & & & & & $<0.01$ \\
\hline Never & $542(15.6 \%)$ & 937 (14.8\%) & $1022(17.8 \%)$ & $559(22.8 \%)$ & - \\
\hline Former & 519 (14.9\%) & $630(9.9 \%)$ & $495(8.6 \%)$ & 245 (10.0\%) & - \\
\hline Current, $<45.6 \mathrm{~g}$ per day ethanol & $1163(33.4 \%)$ & $2684(42.3 \%)$ & $2453(42.8 \%)$ & 968 (39.4\%) & - \\
\hline Current, $\geqslant 45.6 \mathrm{~g}$ per day ethanol & $1255(36.1 \%)$ & $2094(33.0 \%)$ & $1762(30.7 \%)$ & $682(27.8 \%)$ & - \\
\hline Family history of cancer, $n(\%)$ & & & & & 0.86 \\
\hline No & $2574(70.8 \%)$ & $4691(70.2 \%)$ & $4188(70.3 \%)$ & $1829(71.0 \%)$ & - \\
\hline Yes & $1063(29.2 \%)$ & $1989(29.8 \%)$ & $1771(29.7 \%)$ & $748(29.0 \%)$ & - \\
\hline $\begin{array}{l}\text { Total energy intake, mean (s.d.), kcal per } \\
\text { day }\end{array}$ & $1794(596.6)$ & $1820(592.0)$ & $1851(599.5)$ & $1920(607.1)$ & $<0.01$ \\
\hline Daily consumption of miso soup, $n(\%)$ & & & & & $<0.01$ \\
\hline No & $356(9.9 \%)$ & $631(9.6 \%)$ & $674(11.5 \%)$ & $348(13.7 \%)$ & - \\
\hline Yes & $3230(90.1 \%)$ & $5953(90.4 \%)$ & $5201(88.5 \%)$ & $2188(86.3 \%)$ & - \\
\hline Tea consumption, $n(\%)$ & & & & & $<0.01$ \\
\hline$<1$ cups per day & $1183(32.7 \%)$ & $1725(27.9 \%)$ & $1485(28.6 \%)$ & 746 (33.3\%) & - \\
\hline $1-2$ cups per day & 693 (19.2\%) & $1316(21.3 \%)$ & $1526(29.4 \%)$ & $536(23.9 \%)$ & - \\
\hline
\end{tabular}


Table 1. (Continued)

Frequency of coffee consumption

\begin{tabular}{|c|c|c|c|c|c|}
\hline Characteristics & Never $(n=3,637)$ & $\begin{array}{l}\text { Occasionally } \\
(n=6,680)\end{array}$ & $\begin{array}{l}\text { 1-2 Cups per day } \\
(n=5,959)\end{array}$ & $\begin{array}{c}\geqslant 3 \text { Cups per day } \\
(n=2,577)\end{array}$ & $P$-value \\
\hline $3-4$ cups per day & 701 (19.4\%) & 1315 (21.3\%) & 1100 (21.2\%) & 409 (18.3\%) & - \\
\hline$\geqslant 5$ cups per day & $1036(28.7 \%)$ & $1826(29.5 \%)$ & $1076(20.7 \%)$ & $550(24.5 \%)$ & - \\
\hline
\end{tabular}

abtained by using $\chi^{2}$ test for variables of proportion and one-factor ANOVA for continuous variables.

Table 2. The association of coffee consumption with incidence of prostate cancer

Frequency of coffee consumption

\begin{tabular}{|c|c|c|c|c|c|}
\hline & Never & Occasionally & 1-2 Cups per day & $\geqslant 3$ Cups per day & $\boldsymbol{P}_{\text {trend }}$ \\
\hline Total participants (person-years) & 31128.3 & 59029.3 & 54348.8 & 23674.9 & - \\
\hline Prostate cancer (cases) & 84 & 124 & 86 & 24 & - \\
\hline Crude HR (95\% Cl) & 1.00 & $0.77(0.58-1.01)$ & $0.57(0.42-0.77)$ & $0.36(0.23-0.57)$ & $<0.01$ \\
\hline Age-adjusted HR (95\% Cl) & 1.00 & $0.83(0.63-1.10)$ & $0.77(0.57-1.05)$ & $0.65(0.41-1.03)$ & 0.04 \\
\hline Multivariate-adjusted HR1 ${ }^{\text {a }}(95 \% \mathrm{Cl}$ ) & 1.00 & $0.82(0.62-1.08)$ & $0.74(0.54-1.01)$ & $0.63(0.39-1.00)$ & 0.02 \\
\hline Multivariate-adjusted $\mathrm{HR}^{\mathbf{b}}(95 \% \mathrm{Cl})$ & 1.00 & $0.81(0.61-1.07)$ & $0.73(0.53-1.00)$ & $0.63(0.39-1.00)$ & 0.02 \\
\hline Multivariate-adjusted HR2 ${ }^{\mathrm{b}}{ }^{\mathrm{c}}(95 \% \mathrm{Cl})$ & 1.00 & $0.81(0.60-1.08)$ & $0.70(0.50-0.98)$ & $0.68(0.42-1.10)$ & 0.03 \\
\hline
\end{tabular}

${ }^{a_{H R} 1}$ have been adjusted for age (continuous variable), education level (junior high school or lower, high school, college/university or higher), BMI ( $<18.5,18.5-24.9$, or $\geqslant 25.0$ kg m ${ }^{-2}$ ), time engaging in sports or exercise $(<1,1-2$ or $\geqslant 3 \mathrm{~h}$ per week), marital status (married, or widowed/divorced/single), time spent walking ( $<1$ or $\geqslant h$ per day), smoking status (never, former, current smoking $<20$ or current smoking $\geqslant 20$ cigarettes per day), family history of cancer (yes or no), consumption of tea $(<1,1-2,3-4$ or $\geqslant 5$ cups per day).

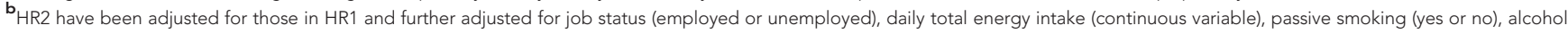
drinking (never, former, current drinking $<45.6$ or current drinking $\geqslant 45.6 \mathrm{~g}$ per day ethanol), daily consumption of miso soup (yes or no).

${ }^{\mathrm{c}}$ Participants who developed cancer within the first 2 years of follow-up were excluded.

with $\geqslant 45.6 \mathrm{~g}$ per day ethanol), and daily consumption of miso soup (yes or no). To ensure that the estimates were not biased by multicollinearity, the unadjusted, age-adjusted, and different multivariate adjusted models were also computed and compared. Interactions between confounders were tested through the addition of cross-product terms to the multivariateadjusted model.

\section{RESULTS}

The detailed characteristics of the subjects according to coffee consumption category are shown in Table 1. Among the participants, 3637 reported that they never drank coffee, 6680 drank coffee only occasionally, 5959 drank 1-2 cups of coffee per day, and 2577 drank $\geqslant 3$ cups per day. Individuals who drank coffee more frequently tended to be younger, with a higher education level, and were more likely to be employed, but tended to walk and engage in sports less frequently. Frequent coffee drinkers were also more likely to be current heavy smokers, to have a greater chance of exposure to passive smoking, to have a higher total calorie intake, and to consume less miso soup. There were no significant relationships of coffee consumption to average BMI, marital status, or presence/absence of a family history of cancer.

A total of 318 cases of prostate cancer were documented during the 11 years of follow-up. Table 2 shows the association between coffee consumption and the incident risk of prostate cancer. Compared with subjects who never drank coffee, the multivariate- adjusted HRs (95\% CIs) were 0.81 (0.61-1.07), 0.73 (0.53-1.00), and $0.63(0.39-1.00)$ for those who drank coffee occasionally, 1-2 cups per day, and $\geqslant 3$ cups per day, respectively ( $P$ for trend, 0.02 ). The HRs were stable when adjusted for age or multi-adjusted for different confounders. The inverse association was also consistent after excluding cases of prostate cancer diagnosed in the first 2 years of follow-up.

Hazard ratios for prostate cancer were recalculated after stratifying the participants by age, BMI level, and smoking status (Table 3). The association between coffee consumption and prostate cancer was statistically significant for men who were older (age $\geqslant 60$ years, $P=0.04$ ), not overweight (BMI $<25 \mathrm{~kg} \mathrm{~m}^{-2}$, $P=0.03)$, and current smokers $(P<0.01)$. There was no interaction between coffee drinking and age, or between coffee drinking and BMI, or between coffee drinking and alcohol drinking. However, for the interaction between coffee drinking and cigarette smoking, a statistically significant interaction was detected $(P=0.03)$.

We further tested the association between coffee consumption and different clinical stages of prostate cancer (Table 4). The HRs for prostate cancer in men who drank coffee most frequently were less than unity for localised stage (multivariate-adjusted HR 0.46), advanced stage (multivariate-adjusted HR 0.77), and missing stage (multivariate-adjusted HR 0.60). This indicated a potentially protective effect of coffee in terms of the risk of prostate cancer for both localised stage and advanced stage, although it did not reach statistical significance.

We analysed the association of coffee consumption with prostate cancer incidence according to differences in the type of coffee/condiments consumed (Table 5). Consumption of sugared 
Table 3. The association of coffee consumption with prostate cancer incidence stratified by age, BMI, and smoking

Frequency of coffee consumption

\begin{tabular}{|c|c|c|c|c|c|c|}
\hline & Never & Occasionally & 1-2 Cups per day & $\geqslant 3$ Cups per day & $\boldsymbol{P}_{\text {trend }}$ & $\boldsymbol{P}_{\text {interaction }}$ \\
\hline Age $<60$ (cases) & 10 & 16 & 15 & 9 & - & 0.21 \\
\hline Age-adjusted HR (95\% Cl) & 1.00 & $0.79(0.36-1.74)$ & $0.71(0.32-1.58)$ & $1.02(0.41-2.52)$ & 0.89 & - \\
\hline Multivariate-adjusted $\mathrm{HR}^{\mathrm{a}}(95 \% \mathrm{Cl})$ & 1.00 & $0.75(0.33-1.67)$ & $0.66(0.29-1.51)$ & $1.07(0.41-2.76)$ & 0.92 & - \\
\hline Multivariate-adjusted $\mathrm{HR}^{\mathrm{a}, \mathrm{b}}(95 \% \mathrm{Cl})$ & 1.00 & $0.75(0.33-1.67)$ & $0.66(0.29-1.51)$ & $1.07(0.41-2.76)$ & 0.92 & - \\
\hline Age $\geqslant 60$ (Cases) & 74 & 108 & 71 & 15 & - & - \\
\hline Age-adjusted HR (95\% Cl) & 1.00 & $0.83(0.62-1.12)$ & $0.80(0.58-1.11)$ & $0.58(0.33-1.01)$ & 0.04 & - \\
\hline Multivariate-adjusted $\mathrm{HR}^{\mathrm{a}}(95 \% \mathrm{Cl})$ & 1.00 & $0.82(0.61-1.11)$ & $0.76(0.54-1.07)$ & $0.55(0.31-0.97)$ & 0.03 & - \\
\hline Multivariate-adjusted $\mathrm{HR}^{\mathrm{a}, \mathrm{b}}(95 \% \mathrm{Cl})$ & 1.00 & $0.82(0.60-1.13)$ & $0.73(0.51-1.05)$ & $0.62(0.35-1.11)$ & 0.04 & - \\
\hline BMI $<25$ (Cases) & 65 & 96 & 62 & 16 & - & 0.17 \\
\hline Age-adjusted HR (95\% Cl) & 1.00 & $0.83(0.60-1.13)$ & $0.72(0.50-1.02)$ & $0.55(0.32-0.96)$ & 0.02 & - \\
\hline Multivariate-adjusted $\mathrm{HR}^{\mathrm{a}}(95 \% \mathrm{Cl})$ & 1.00 & $0.83(0.60-1.14)$ & $0.70(0.49-1.01)$ & $0.58(0.33-1.02)$ & 0.02 & - \\
\hline Multivariate-adjusted $\mathrm{HR}^{\mathrm{a}, \mathrm{b}}(95 \% \mathrm{Cl})$ & 1.00 & $0.85(0.60-1.19)$ & $0.66(0.45-0.98)$ & $0.64(0.36-1.15)$ & 0.03 & - \\
\hline $\mathrm{BMI} \geqslant 25$ (Cases) & 16 & 23 & 18 & 7 & - & - \\
\hline Age-adjusted HR (95\% Cl) & 1.00 & $0.82(0.43-1.56)$ & $0.86(0.44-1.70)$ & $1.05(0.43-2.59)$ & 0.97 & - \\
\hline Multivariate-adjusted $\mathrm{HR}^{\mathrm{a}}(95 \% \mathrm{Cl})$ & 1.00 & $0.73(0.38-1.41)$ & $0.75(0.37-1.52)$ & $0.89(0.35-2.26)$ & 0.67 & - \\
\hline Multivariate-adjusted $\mathrm{HR}^{\mathrm{a}, \mathrm{b}}(95 \% \mathrm{Cl})$ & 1.00 & $0.69(0.35-1.34)$ & $0.72(0.35-1.48)$ & $0.94(0.37-2.41)$ & 0.73 & - \\
\hline Non-smoker or past smoker (Cases) & 45 & 67 & 43 & 11 & & 0.03 \\
\hline Age-adjusted HR (95\% Cl) & 1.00 & $0.91(0.63-1.33)$ & $0.89(0.59-1.36)$ & $0.96(0.49-1.86)$ & 0.70 & - \\
\hline Multivariate-adjusted $\mathrm{HR}^{\mathrm{a}}(95 \% \mathrm{Cl})$ & 1.00 & $0.88(0.60-1.29)$ & $0.86(0.56-1.33)$ & $0.94(0.48-1.85)$ & 0.62 & - \\
\hline Multivariate-adjusted $\mathrm{HR}^{\mathrm{a}, \mathrm{b}}(95 \% \mathrm{Cl})$ & 1.00 & $0.83(0.55-1.23)$ & $0.79(0.50-1.24)$ & $0.98(0.50-1.94)$ & 0.54 & - \\
\hline Current smoker (Cases) & 37 & 52 & 39 & 11 & & - \\
\hline Age-adjusted HR (95\% Cl) & 1.00 & $0.74(0.48-1.12)$ & $0.63(0.40-0.99)$ & $0.43(0.22-0.84)$ & 0.01 & - \\
\hline Multivariate-adjusted $\mathrm{HR}^{\mathrm{a}}(95 \% \mathrm{Cl})$ & 1.00 & $0.70(0.46-1.08)$ & $0.58(0.36-0.93)$ & $0.37(0.18-0.75)$ & $<0.01$ & - \\
\hline Multivariate-adjusted $\mathrm{HR}^{\mathrm{a}, \mathrm{b}}(95 \% \mathrm{Cl})$ & 1.00 & $0.72(0.46-1.13)$ & $0.58(0.35-0.95)$ & $0.41(0.20-0.85)$ & $<0.01$ & - \\
\hline Non-alocohol drinker (Cases) & 60 & 84 & 58 & 17 & - & 0.35 \\
\hline Age-adjusted HR (95\% Cl) & 1.00 & $0.69(0.50-0.96)$ & $0.52(0.36-0.75)$ & $0.39(0.23-0.66)$ & $<0.01$ & - \\
\hline Multivariate-adjusted $\mathrm{HR}^{\mathrm{a}}(95 \% \mathrm{Cl})$ & 1.00 & $0.76(0.55-1.06)$ & $0.75(0.52-1.08)$ & $0.77(0.45-1.33)$ & 0.18 & - \\
\hline Multivariate-adjusted $\mathrm{HR}^{\mathrm{a}, \mathrm{b}}(95 \% \mathrm{Cl})$ & 1.00 & $0.77(0.54-1.09)$ & $0.64(0.43-0.96)$ & $0.79(0.45-1.39)$ & 0.10 & - \\
\hline Alocohol drinker (cases) & 23 & 38 & 26 & 5 & - & - \\
\hline Age-adjusted HR (95\% Cl) & 1.00 & $1.03(0.61-1.73)$ & $0.69(0.40-1.22)$ & $0.24(0.09-0.63)$ & $<0.01$ & - \\
\hline Multivariate-adjusted $\mathrm{HR}^{\mathrm{a}}(95 \% \mathrm{Cl})$ & 1.00 & $1.06(0.63-1.78)$ & $0.82(0.47-1.44)$ & $0.38(0.14-1.00)$ & 0.05 & - \\
\hline Multivariate-adjusted $\mathrm{HR}^{\mathbf{a}, \mathbf{b}}(95 \% \mathrm{Cl}$ ) & 1.00 & $1.13(0.66-1.91)$ & $0.88(0.49-1.58)$ & $0.39(0.14-1.08)$ & 0.10 & - \\
\hline First half of follow-up (Cases) & 29 & 37 & 30 & 7 & - & - \\
\hline Age-adjusted HR (95\% Cl) & 1.00 & $0.68(0.42-1.11)$ & $0.61(0.36-1.01)$ & $0.33(0.14-0.75)$ & $<0.01$ & - \\
\hline Multivariate-adjusted $\mathrm{HR}^{\mathrm{a}}(95 \% \mathrm{Cl})$ & 1.00 & $0.73(0.44-1.19)$ & $0.85(0.50-1.44)$ & $0.68(0.29-1.60)$ & 0.45 & - \\
\hline First half of follow-up (Cases) & 55 & 87 & 56 & 17 & - & - \\
\hline Age-adjusted HR (95\% Cl) & 1.00 & $0.81(0.58-1.14)$ & $0.55(0.38-0.80)$ & $0.38(0.22-0.65)$ & $<0.01$ & - \\
\hline Multivariate-adjusted $\mathrm{HR}^{\mathrm{a}}(95 \% \mathrm{Cl})$ & 1.00 & $0.85(0.60-1.19)$ & $0.67(0.46-1.00)$ & $0.59(0.33-1.04)$ & 0.02 & - \\
\hline
\end{tabular}

${ }^{a_{H R}}$ have been adjusted for age (continuous variable), education level (junior high school or lower, high school, college/university or higher), BMI ( $<18.5,18.5-24.9$, or $\geqslant 25.0 \mathrm{~kg} \mathrm{~m}{ }^{-2}$ ), time engaging in sports or exercise ( $<1,1-2$ or $\geqslant 3 \mathrm{~h}$ per week), marital status (married, or widowed/divorced/single), time spent walking ( $<1$ or $\geqslant 1 \mathrm{~h}$ per day), smoking status (never, former, current smoking $<20$ or current smoking $\geqslant 20$ cigarettes per day), family history of cancer (yes or no), consumption of tea ( $<1,1-2,3-4$ or $\geqslant 5$ cups per day), job status (employed or unemployed), daily total energy intake (continuous variable), passive smoking (yes or no), alcohol drinking (never, former, current drinking $<45.6$ or current drinking $\geqslant 45.6 \mathrm{~g}$ per day ethanol), daily consumption of miso soup (yes or no)

b Participants who developed cancer within the first 2 years of follow-up were excluded.

coffee and sugar-free coffee showed a similar relationship with prostate cancer risk, the point-estimated HRs being close. The significant $P$-value in the group who drank sugared coffee may have been attributable to the larger sample size, as most people in the study area added sugar to coffee. Only consumption of coffee without milk or cream appeared to afford significant protection against prostate cancer. The HR was 0.42 (95\% CI: $0.21-0.85)$ for subjects who frequently drank coffee without added milk or cream $v s$ non-coffee-drinkers, whereas it was 0.81 (95\% CI: 0.46-1.44) for those who frequently drank coffee with added milk or cream $v s$ 
Table 4. The association of coffee consumption with prostate cancer incidence

Frequency of coffee consumption

\begin{tabular}{|c|c|c|c|c|c|}
\hline Stage & Never & Occasionally & 1-2 Cups per day & $\geqslant 3$ Cups per day & $\boldsymbol{P}_{\text {trend }}$ \\
\hline Localised stage (cases) & 18 & 29 & 27 & 4 & \\
\hline Age-adjusted HR $(95 \% \mathrm{Cl})$ & 1.00 & $0.91(0.50-1.63)$ & $1.13(0.62-2.05)$ & $0.50(0.17-1.50)$ & 0.65 \\
\hline Multivariate-adjusted $\mathrm{HR}^{\mathrm{a}}(95 \% \mathrm{Cl})$ & 1.00 & $0.86(0.47-1.56)$ & $1.08(0.58-2.00)$ & $0.46(0.15-1.41)$ & 0.54 \\
\hline Multivariate-adjusted $\mathrm{HR}^{\mathbf{b}}(95 \% \mathrm{Cl})$ & 1.00 & $0.89(0.48-1.65)$ & $1.16(0.61-2.20)$ & $0.54(0.18-1.66)$ & 0.77 \\
\hline Advanced stage (cases) & 24 & 50 & 27 & 8 & - \\
\hline Age-adjusted HR (95\% Cl) & 1.00 & $1.18(0.73-1.93)$ & $0.86(0.49-1.49)$ & $0.76(0.34-1.71)$ & 0.32 \\
\hline Multivariate-adjusted $\mathrm{HR}^{\mathrm{a}}(95 \% \mathrm{Cl})$ & 1.00 & $1.21(0.74-1.99)$ & $0.89(0.50-1.57)$ & $0.77(0.34-1.78)$ & 0.38 \\
\hline Multivariate-adjusted $\mathrm{HR}^{\mathbf{b}}(95 \% \mathrm{Cl})$ & 1.00 & $1.26(0.73-2.16)$ & $0.73(0.38-1.39)$ & $0.90(0.38-2.12)$ & 0.33 \\
\hline Missing stage (cases) & 42 & 45 & 32 & 12 & - \\
\hline Age-adjusted HR (95\% Cl) & 1.00 & $0.60(0.39-0.92)$ & $0.57(0.36-0.91)$ & $0.65(0.34-1.24)$ & 0.05 \\
\hline Multivariate-adjusted $\mathrm{HR}^{\mathrm{a}}(95 \% \mathrm{Cl})$ & 1.00 & $0.57(0.37-0.87)$ & $0.50(0.31-0.81)$ & $0.60(0.31-1.18)$ & 0.02 \\
\hline Multivariate-adjusted $\mathrm{HR}^{\mathbf{b}}(95 \% \mathrm{Cl})$ & 1.00 & $0.55(0.35-0.85)$ & $0.50(0.30-0.81)$ & $0.61(0.31-1.20)$ & 0.03 \\
\hline
\end{tabular}

Table 5. The association of coffee consumption with prostate cancer incidence in different coffee type

Frequency of coffee consumption

\begin{tabular}{|c|c|c|c|c|c|}
\hline & Never & Occasionally & 1-2 Cups per day & $\geqslant 3$ Cups per day & $\boldsymbol{P}_{\text {trend }}$ \\
\hline Coffee without sugar (cases) & 84 & 33 & 23 & 8 & - \\
\hline Age-adjusted HR (95\% Cl) & 1.00 & $0.71(0.48-1.07)$ & $0.72(0.45-1.15)$ & $0.68(0.33-1.42)$ & 0.08 \\
\hline Multivariate-adjusted $\mathrm{HR}^{\mathrm{a}}(95 \% \mathrm{Cl})$ & 1.00 & $0.69(0.46-1.05)$ & $0.67(0.41-1.10)$ & $0.67(0.31-1.43)$ & 0.07 \\
\hline Coffee with sugar (cases) & 84 & 91 & 63 & 16 & - \\
\hline Age-adjusted HR (95\% Cl) & 1.00 & $0.88(0.66-1.19)$ & $0.79(0.57-1.10)$ & $0.63(0.37-1.08)$ & 0.05 \\
\hline Multivariate-adjusted $\mathrm{HR}^{\mathrm{a}}(95 \% \mathrm{Cl})$ & 1.00 & $0.85(0.63-1.15)$ & $0.73(0.52-1.02)$ & $0.57(0.33-0.99)$ & 0.02 \\
\hline Coffee without milk or cream (cases) & 84 & 60 & 34 & 9 & - \\
\hline Age-adjusted HR (95\% Cl) & 1.00 & $0.75(0.54-1.05)$ & $0.64(0.43-0.95)$ & $0.44(0.22-0.88)$ & $<0.01$ \\
\hline Multivariate-adjusted $\mathrm{HR}^{\mathrm{a}}(95 \% \mathrm{Cl})$ & 1.00 & $0.72(0.52-1.02)$ & $0.60(0.39-0.91)$ & $0.42(0.21-0.85)$ & $<0.01$ \\
\hline Coffee with milk or cream (cases) & 84 & 64 & 52 & 15 & - \\
\hline Age-adjusted HR $(95 \% \mathrm{Cl})$ & 1.00 & $0.91(0.66-1.27)$ & $0.89(0.63-1.25)$ & $0.88(0.51-1.54)$ & 0.48 \\
\hline Multivariate-adjusted $\mathrm{HR}^{\mathrm{a}}(95 \% \mathrm{Cl})$ & 1.00 & $0.89(0.64-1.25)$ & $0.83(0.58-1.19)$ & $0.81(0.46-1.44)$ & 0.29 \\
\hline
\end{tabular}

non-coffee-drinkers. No interaction between coffee and sugar, or between coffee and milk, was found.

We also investigated the relationship between other individual caffeine-containing beverages and the risk of prostate cancer. However, no association with the risk of prostate cancer was found for the consumption of green tea, black tea or oolong tea (Table 6).

\section{DISCUSSION}

In this population-based, prospective cohort that included 18853 Japanese men among whom 318 cases of prostate cancer were detected during an 11-year follow-up, we observed an inverse association between coffee consumption and the incident risk of prostate cancer. The protective effects of coffee drinking were robust in men who were older, non-overweight, and current smokers. A dose-response relationship was observed, as $\sim 37 \%$ of prostate cancers were estimated to be prevented in men consuming three or more cups of coffee per day, compared with non-coffee drinkers.

It remains unclear which constituent(s) of coffee contributed to the protective affect against prostate cancer. Caffeine, the major component of coffee, has been thought to inhibit carcinogenesis in vivo (Hashimoto et al, 2004). Green tea, black tea and oolong tea also contain caffeine, but we did not find any protective effect of these beverages against prostate cancer. Therefore, we consider that caffeine does not have a role in protecting against prostate cancer. The coffee-specific diterpenes, cafestol and kahweol, have been proved to possess anticarcinogenetic activity (Cavin et al, 2002). Furthermore, coffee drinking may be associated with increased 


\begin{tabular}{|c|c|c|c|c|c|}
\hline & \multicolumn{4}{|c|}{ Frequency of consumption } & \multirow[b]{2}{*}{$\boldsymbol{P}_{\text {trenc }}$} \\
\hline & Never & Occasionally & 1-2 Cups perDay & $\geqslant 3$ Cups per day & \\
\hline \multicolumn{6}{|l|}{ Green tea } \\
\hline $\begin{array}{l}\text { Prostate cancer (cases) } \\
\text { Following period (person-years) } \\
\text { Age-adjusted HR ( } 95 \% \mathrm{Cl}) \\
\text { Multivariate-adjusted } \mathrm{HR}^{\mathrm{a}}(95 \% \mathrm{Cl})\end{array}$ & $\begin{array}{c}17 \\
14520 \\
1.00 \\
1.00\end{array}$ & $\begin{array}{c}55 \\
34236 \\
1.22(0.83-1.80) \\
1.18(0.80-1.75)\end{array}$ & $\begin{array}{c}56 \\
34416 \\
1.17(0.80-1.72) \\
1.12(0.76-1.66)\end{array}$ & $\begin{array}{c}159 \\
68821 \\
1.32(0.96-1.82) \\
1.26(0.90-1.76)\end{array}$ & $\begin{array}{l}- \\
- \\
0.58 \\
0.37\end{array}$ \\
\hline \multicolumn{6}{|l|}{ Black tea } \\
\hline $\begin{array}{l}\text { Prostate cancer (cases) } \\
\text { Following period (Person-years) } \\
\text { Age-adjusted } \mathrm{HR}(95 \% \mathrm{Cl}) \\
\text { Multivariate-adjusted } \mathrm{HR}^{\mathrm{a}}(95 \% \mathrm{Cl})\end{array}$ & $\begin{array}{c}168 \\
87488 \\
1.00 \\
1.00\end{array}$ & $\begin{array}{c}66 \\
41247 \\
0.94(0.71-1.23) \\
0.88(0.67-1.17)\end{array}$ & $\begin{array}{c}6 \\
3110 \\
0.97(0.43-2.18) \\
0.93(0.41-2.10)\end{array}$ & $\begin{array}{c}2 \\
1008 \\
0.92(0.23-3.69) \\
0.94(0.23-3.81)\end{array}$ & $\begin{array}{l}- \\
- \\
0.72 \\
0.95\end{array}$ \\
\hline \multicolumn{6}{|l|}{ Oolong tea } \\
\hline $\begin{array}{l}\text { Prostate cancer (cases) } \\
\text { Following period (person-years) } \\
\text { Age-adjusted HR ( } 95 \% \mathrm{Cl}) \\
\text { Multivariate-adjusted } \mathrm{HR}^{\mathrm{a}}(95 \% \mathrm{Cl})\end{array}$ & $\begin{array}{c}155 \\
82890 \\
1.00 \\
1.00\end{array}$ & $\begin{array}{c}62 \\
39567 \\
0.94(0.71-1.24) \\
0.90(0.68-1.20)\end{array}$ & $\begin{array}{c}11 \\
7026 \\
1.07(0.58-1.95) \\
1.03(0.56-1.89)\end{array}$ & $\begin{array}{c}10 \\
4652 \\
1.26(0.67-2.37) \\
1.18(0.63-2.24)\end{array}$ & $\begin{array}{l}- \\
- \\
0.61 \\
0.31\end{array}$ \\
\hline
\end{tabular}

levels of sex hormone-binding globulin and testosterone, which have a role in prostate cancer (Svartberg et al, 2003). Coffee consumption is also associated with reductions in the levels of inflammation-related molecules, which may have a potential role in prostatic carcinogenesis and tumour progression (Maki et al, 2010; Sfanos and De Marzo, 2012).

The protective effect against prostate cancer was more robust in subjects who drank coffee without added milk or cream than in those who added milk or cream. As milk consumption has been shown to be positively associated with prostate cancer risk in previous case-control and cohort studies (Qin et al, 2004, 2007), it is reasonable that drinking coffee with added milk or cream would afford less-effective protection. Higher risk of prostate cancer incidence was found for current smokers, and the protective effects of coffee on prostate cancer were also stronger for current smokers with interaction between coffee and smoking being found.

Existing reviews of the epidemiological literature reveal inconsistencies in the effects of coffee consumption on prostate cancer, although they may have been methodologically flawed (Arab, 2011). One meta-analysis published in 2010 found no such association in cohort studies (Park et al, 2010), while another metaanalysis published in 2011 demonstrated a protective effect of coffee based on five prospective cohort studies (Yu et al, 2011). Our results are coincident with the second meta-analysis, and are also generally consistent with the recent large cohort study from USA and the small cohort conducted in UK (Wilson et al, 2011; Shafique et al, 2012). The cohort study in USA found a protective effect in subjects who drank six or more cups of coffee daily $v s$ non-coffee-drinkers, and the effect was more significant for advanced or lethal prostate cancer (Wilson et al, 2011). The investigation in UK suggested that consumption of three or more cups of coffee daily had the greatest effect $v s$ non-coffee-drinkers in terms of high Gleason grade prostate cancer (Shafique et al, 2012). Our significant dose-response result from an Asian population has contributed new evidence of coffee's effect on overall prostate cancer. The discrepancy in findings between the present study and earlier cohort studies may be partially attributed to the limited number of incident cases of prostate cancer, and inadequate adjustment for covariates (Phillips and Snowdon, 1983; Jacobsen et al, 1986; Nomura et al, 1986; Severson et al, 1989; Hsing et al, 1990; Le Marchand et al, 1994; Ellison, 2000; Nilsson et al, 2010).

Prostate specific antigen (PSA) mass screening has been introduced to detect prostate cancer at an early stage. In Japan, PSA mass screening has been provided free (or at low charge) during annual health checkups by public health care facilities to all men aged $\geqslant 40$ once in every 5 years. In our study, the rate of participation in annual health checkups did not differ between subjects of different coffee consumption category. Therefore, our results were less likely to be influenced by PSA mass screening.

Our study had some strengths. The FFQ we used had a reasonably high level of reproducibility and validity, and the elderly population we examined tended to maintain relatively stable dietary habits. We followed up this population for a sufficiently long period and accumulated an adequate number of incident cases of prostate cancer. Furthermore, we tested associations by multivariate analysis in which possible risk factors were carefully considered, and different covariate-adjusted models were employed and compared. In addition, we also stratified the analysis by age, BMI, smoking status, and stage of prostate cancer to control for major confounding factors.

Our study also had several limitations. First, as coffee consumption habits were self-reported and collected only once at the baseline, some misclassification caused by changes in coffee consumption during the follow-up period may have arisen. However, this misclassification would have tended to be nondifferential, resulting in a conservative estimation of the effect of coffee consumption. Second, we did not enquire about the specific types of coffee consumed or the method of preparation, but included all kinds of coffee as a whole. Any potentially cancerrelated chemical ingredients may differ according to the type of coffee consumed (such as decaffeinated or regular coffee) and the preparation method employed (such as filtering or percolating). Third, we excluded 4162 subjects for whom data on coffee consumption were missing, or who reported extreme daily energy intakes (upper $0.5 \%$ and lower 0.5\%). Among the excluded subjects, 74 cases of prostate cancer were diagnosed, which was close to the proportion of subjects involved in our analysis, and the corresponding multivariate-adjusted HR revealed 
no difference in prostate cancer incidence between those included and those excluded $(\mathrm{HR}=0.93, P=0.60)$. Thus, our results would not have been markedly biased by this exclusion. Fourth, our result results showed only an association, and the associations were borderline especially for the stratified analysis. A direct cause-and-effect link between coffee intake and prostate cancer is far from proven.

In conclusion, evidence derived from this population-based prospective cohort study carried out in Japan suggests a protective association between coffee consumption and the risk of prostate cancer. These results indicate that coffee consumption may contribute to the prevention of prostate cancer, and randomized controlled trials are needed to further prove this association.

\section{ACKNOWLEDGEMENTS}

This study was supported by Grant-in-Aid for Cancer Research and for the Third Term Comprehensive Ten-Year Strategy for Cancer Control, Ministry of Health, Labour and Welfare, Japan (grants H23-Junkankitou (Seisyuu)-Ippan-005). We thank China Scholarship Council offering scholarship to Qiang Li to conduct this research in Japan.

\section{CONFLICT OF INTEREST}

The authors declare no confilict of interest.

\section{DISCLAIMER}

The funding body has no role in study design; in the data collection, analysis, and interpretation; in the writing of the report; and in the decision to submit the paper for publication.

\section{REFERENCES}

Arab L (2011) Should men drink more coffee to delay progression of prostate cancer? Nutr Cancer 63(8): 1161-1162.

Cardenas C, Quesada AR, Medina MA (2011) Anti-angiogenic and anti-inflammatory properties of kahweol, a coffee diterpene. PLoS One 6(8): e23407.

Cavin C, Holzhaeuser D, Scharf G, Constable A, Huber WW, Schilter B (2002) Cafestol and kahweol, two coffee specific diterpenes with anticarcinogenic activity. Food Chem Toxicol 40(8): 1155-1163.

Curado MP, Edwards B, Shin HR, Storm H, Ferlay J, Heanue M, Boyle P (2007) Cancer Incidence in Five Continents Vol. IXIARC Scientific Publications: Lyon, (No. 160).

Ellison LF (2000) Tea and other beverage consumption and prostate cancer risk: a Canadian retrospective cohort study. Eur J Cancer Prev 9(2): $125-130$.

Gomez-Ruiz JA, Leake DS, Ames JM (2007) In vitro antioxidant activity of coffee compounds and their metabolites. J Agric Food Chem 55(17): 6962-6969.

Hashimoto T, He Z, Ma WY, Schmid PC, Bode AM, Yang CS, Dong Z (2004) Caffeine inhibits cell proliferation by G0/G1 phase arrest in JB6 cells. Cancer Res 64(9): 3344-3349.

Hsieh CC, Signorello LB, Lipworth L, Lagiou P, Mantzoros CS, Trichopoulos D (1998) Predictors of sex hormone levels among the elderly: a study in Greece. J Clin Epidemiol 51(10): 837-841.

Hsing AW, McLaughlin JK, Schuman LM, Bjelke E, Gridley G, Wacholder S, Chien HT, Blot WJ (1990) Diet, tobacco use, and fatal prostate cancer: results from the Lutheran Brotherhood Cohort Study. Cancer Res 50(21): 6836-6840.

Jacobsen BK, Bjelke E, Kvale G, Heuch I (1986) Coffee drinking, mortality, and cancer incidence: results from a Norwegian prospective study. J Natl Cancer Inst 76(5): 823-831.
Japan Ministry of Health Labour and Welfare (2010) Annual Report of National Health and Nutrition Survey. http://www.mhlw.go.jp/bunya/ kenkou/kenkou_eiyou_chousa.html. (In Japanese; accessed 3 September 2012).

Jemal A, Bray F, Center MM, Ferlay J, Ward E, Forman D (2011) Global cancer statistics. CA Cancer J Clin 61(2): 69-90.

Kuriyama S, Shimazu T, Ohmori K, Kikuchi N, Nakaya N, Nishino Y, Tsubono Y, Tsuji I (2006) Green tea consumption and mortality due to cardiovascular disease, cancer, and all causes in Japan: the Ohsaki study. JAMA 296(10): 1255-1265.

Le Marchand L, Kolonel LN, Wilkens LR, Myers BC, Hirohata T (1994) Animal fat consumption and prostate cancer: a prospective study in Hawaii. Epidemiology 5(3): 276-282.

Li Q, Kakizaki M, Kuriyama S, Sone T, Yan H, Nakaya N, Mastuda-Ohmori $\mathrm{K}$, Tsuji I (2008) Green tea consumption and lung cancer risk: the Ohsaki study. Br J Cancer 99(7): 1179-1184.

Maki T, Pham NM, Yoshida D, Yin G, Ohnaka K, Takayanagi R, Kono S (2010) The relationship of coffee and green tea consumption with highsensitivity C-reactive protein in Japanese men and women. Clin Chem Lab Med 48(6): 849-854.

Matsuda T, Marugame T, Kamo K, Katanoda K, Ajiki W, Sobue T. Japan Cancer Surveillance Research Group (2012) Cancer incidence and incidence rates in Japan in 2006: based on data from 15 population-based cancer registries in the monitoring of cancer incidence in Japan (MCIJ) project. Jpn J Clin Oncol 42(2): 139-147.

Naganuma T, Kuriyama S, Kakizaki M, Sone T, Nakaya N, Ohmori-Matsuda K, Nishino Y, Fukao A, Tsuji I (2008) Coffee consumption and the risk of oral, pharyngeal, and esophageal cancers in Japan: the Miyagi Cohort Study. Am J Epidemiol 168(12): 1425-1432.

Nilsson LM, Johansson I, Lenner P, Lindahl B, Van Guelpen B (2010) Consumption of filtered and boiled coffee and the risk of incident cancer: a prospective cohort study. Cancer Causes Control 21(10): 1533-1544.

Nomura A, Heilbrun LK, Stemmermann GN (1986) Prospective study of coffee consumption and the risk of cancer. J Natl Cancer Inst 76(4): 587-590.

Park CH, Myung SK, Kim TY, Seo HG, Jeon YJ, Kim Y (2010) Coffee consumption and risk of prostate cancer: a meta-analysis of epidemiological studies. BJU Int 106(6): 762-769.

Phillips RL, Snowdon DA (1983) Association of meat and coffee use with cancers of the large bowel, breast, and prostate among Seventh-Day Adventists: preliminary results. Cancer Res 43(5 Suppl): 2403s-2408s.

Qin LQ, Xu JY, Wang PY, Kaneko T, Hoshi K, Sato A (2004) Milk consumption is a risk factor for prostate cancer: meta-analysis of casecontrol studies. Nutr Cancer 48(1): 22-27.

Qin LQ, Xu JY, Wang PY, Tong J, Hoshi K (2007) Milk consumption is a risk factor for prostate cancer in Western countries: evidence from cohort studies. Asia Pac J Clin Nutr 16(3): 467-476.

Severson RK, Nomura AM, Grove JS, Stemmermann GN (1989) A prospective study of demographics, diet, and prostate cancer among men of Japanese ancestry in Hawaii. Cancer Res 49(7): 1857-1860.

Sfanos KS, De Marzo AM (2012) Prostate cancer and inflammation: the evidence. Histopathology 60(1): 199-215.

Shafique K, McLoone P, Qureshi K, Leung H, Hart C, Morrison D (2012) Coffee consumption and prostate cancer risk: further evidence for inverse relationship. Nutr J 11(1): 42.

Shimazu T, Tsubono Y, Kuriyama S, Ohmori K, Koizumi Y, Nishino Y, Shibuya D, Tsuji I (2005) Coffee consumption and the risk of primary liver cancer: pooled analysis of two prospective studies in Japan. Int $J$ Cancer 116(1): 150-154.

Svartberg J, Midtby M, Bonaa KH, Sundsfjord J, Joakimsen RM, Jorde R (2003) The associations of age, lifestyle factors and chronic disease with testosterone in men: the Tromso Study. Eur J Endocrinol 149(2): 145-152.

Svilaas A, Sakhi AK, Andersen LF, Svilaas T, Strom EC, Jacobs Jr. DR, Ose L, Blomhoff R (2004) Intakes of antioxidants in coffee, wine, and vegetables are correlated with plasma carotenoids in humans. J Nutr 134(3): 562-567.

Tabata N, Ohno Y, Matsui R, Sugiyama H, Ito Y, Tsukuma H, Oshima A (2008) Partial cancer prevalence in Japan up to 2020: estimates based on incidence and survival data from population-based cancer registries. Jpn J Clin Oncol 38(2): 146-157.

Takano A, Okuno Y (1997) In: Cancer incidence in five continents, Parkin DM, Whelan SL, Ferlay J, Raymond L, Young J (eds.) Vol. 7: 386-389. IARC Sci Publ: Miyagi Prefecture, Japan.

Tsuji I, Nishino Y, Ohkubo T, Kuwahara A, Ogawa K, Watanabe Y, Tsubono Y, Bando T, Kanemura S, Izumi Y, Sasaki A, Fukao A, Nishikori M, 
Hisamichi S (1998) A prospective cohort study on National Health Insurance beneficiaries in Ohsaki, Miyagi Prefecture, Japan: study design, profiles of the subjects and medical cost during the first year. J Epidemiol 8(5): 258-263.

Watanabe I, Kuriyama S, Kakizaki M, Sone T, Ohmori-Matsuda K, Nakaya N, Hozawa A, Tsuji I (2009) Green tea and death from pneumonia in Japan: the Ohsaki cohort study. Am J Clin Nutr 90(3): 672-679.

Wilson KM, Kasperzyk JL, Rider JR, Kenfield S, van Dam RM, Stampfer MJ, Giovannucci E, Mucci LA (2011) Coffee consumption and prostate cancer risk and progression in the Health Professionals Follow-up Study. J Natl Cancer Inst 103(11): 876-884.
World Health Organization (2008) World cancer Report. International Agency for Research on Cancer: Lyon.

Yu X, Bao Z, Zou J, Dong J (2011) Coffee consumption and risk of cancers: a meta-analysis of cohort studies. BMC Cancer 11: 96.

This work is published under the standard license to publish agreement. After 12 months the work will become freely available and the license terms will switch to a Creative Commons AttributionNonCommercial-Share Alike 3.0 Unported License. 